\title{
THE TYCHO CATALOGUE: EXTENSION OF OPTICAL REFERENCE FRAME
}

\author{
E. HøG \\ Copenhagen University Observatory \\ Juliane Maries Vej 30, DK-2100 Copenhagen Ø, Denmark
}

\begin{abstract}
The Tycho Catalogue contains astrometric and photometric data for the one million brightest stars on the sky. About 900000 stars are high quality astrometric reference stars. Photometry in a blue and visual band is provided.

Two extensions of the catalogue are under way. One is the extension to one magnitude fainter stars by a second reduction of the original Tycho observations, resulting in a catalogue with a total of about three million stars. The other extension is the derivation of more accurate proper motions by means of the Astrographic Catalogue positions as first epoch. This latter work will be carried out first for the one million stars, and later for all the three million, resulting in the Tycho Reference Catalogue (TRC).
\end{abstract}

\section{The Tycho Catalogue (TYC)}

The Tycho Catalogue contains astrometric and photometric data for the one million brightest stars in the sky, based on observations with the Hipparcos satellite star mapper. The median astrometric standard errors (in position, parallax, and annual proper motion) are typically around 7 mas for stars brighter than $V_{T} \sim 9 \mathrm{mag}$, and approximately 25 mas for $V_{T} \sim 10.5 \mathrm{mag}$, at the catalogue epoch (J1991.25). The regional systematic errors are $\sim 1 \operatorname{mas}(/ \mathrm{yr})$. The catalogue is available in machine-readable form as part of the final Hipparcos mission products (ESA 1997), including a detailed description of the Tycho Catalogue contents (in Volume 1) and construction (in Volume 4). Shorter descriptions are given by Høg (1997a, 1997b) and Høg et al. (1997).

\section{The second Tycho Catalogue (TYC2)}

The original Tycho observations comprising 1000 Gbits of data are now subject to a second processing (see Høg 1997a, Section 6). This should result in a second Tycho Catalogue (TYC 2) of 3 million stars, including the one million in the present Tycho Catalogue. Expected standard errors at $\mathrm{V}=11.0$ and 12.0 are respectively about 50 and 100 mas for the position components, and 0.10 and 0.20 mag for visual magnitudes. The reduction is being carried out at Copenhagen in collaboration with Heidelberg, with planned completion in 1999.

The second Tycho processing of all raw counts is based on the available satellite attitude, the Tycho Catalogue of one million stars, and a newly constructed Tycho Input Catalogue (TIC2) of 4.3 million stars. The TIC2 production made use of a new astrometric reduction of GSC (GSC1.2 by Röser et al. 1996) and the Astrographic Catalogue. The USNO A1.0 catalogue (Monet et al. 1996) delivered many of the positions, proper motions and colour indices. The TIC2 is as complete as possible to about $V=12.5 \mathrm{mag}$ and has a position accuracy about 0.25 arcsec at the observation epoch. The magnitudes in TIC2 obtained from GSC have been corrected by means of the Tycho Catalogue photometry (Fabricius 1997). The processing is expected to give better astrometric and photometric values for the fainter half of the present one million stars of the Tycho Catalogue. The major reason is that an astrometric estimation based on very few photons does not achieve the Cramér-Rao limit, cf. Yoshizawa \& Andreasen \& Høg (1985). An estimation based on a superpositioning of all photons from many transits will come much closer to that limit. Furthermore, many 
transits of the faint stars were below the limit of signal-to-noise ratio 1.5 used for the detection in the first Tycho reduction and were thus completely lost.

\section{Tycho Reference Catalogue (TRC)}

The Tycho Catalogue constitutes an astrometric reference frame with twice as many stars as PPM (Röser \& Bastian 1991) which is the best frame up to now with high star density, cf. Table 2 by Høg (1995). The 30 mas standard error of positions for TYC at the epoch 1990 is ten times smaller than that of PPM and the systematic errors are 100 times smaller. The accuracy is maintained for one or two decades from 1990 if PPM proper motions are used. Even better proper motions will be derived for all Tycho stars by means of positions from the Astrographic Catalogue, reduced to the Hipparcos reference system. This Tycho Reference Catalogue project was proposed by Röser \& Høg (1993) and pilot project results are described by Kuzmin et al. (1997). The expected standard errors of the proper motion components are about $2.5 \mathrm{mas} / \mathrm{yr}$.

The Astrographic Catalogue will be transferred to the Hipparcos reference system using the PPM catalogue, the ACRS (Corbin \& Urban 1991) and the Hipparcos and Tycho Catalogues. A direct use of the Hipparcos Catalogue only is not feasible due to the small number of Hipparcos reference stars per plate. Therefore, the much more numerous Tycho Catalogue stars are better suited to derive certain systematic plate-specific errors of the Astrographic Catalogue even though the Tycho Catalogue proper motions are too inaccurate to transfer the positions properly to the AC epoch. This work is being carried out at Moscow, in collaboration with Copenhagen, Heidelberg and Lund. A first preliminary version of TRC with one million stars should appear in late 1997. After completion of the second Tycho processing a TRC with 3 million stars will be produced.

\section{References}

Corbin, T.E., Urban, S.E. 1991, Astrographic Catalogue Reference Stars, U.S. Naval Observatory, Washington

ESA 1997, The Hipparcos and Tycho Catalogues, ESA SP-1200

Fabricius, C. 1997, in Proceedings of the HIPPARCOS Venice '97 Symposium, ESA SP-402

Høg E. 1995, in: E. Høg and P.K. Seidelmann (eds.), Astronomical and Astrophysical Objectives of Sub-Milliarcsecond Optical Astrometry, p. 317, Kluwer Academic Publ., IAU Symposium No. 166

Høg, E. 1997a, The Tycho Catalogue: Astrometry and Photometry, in Proceedings of the HIPPARCOS Venice ' 97 Symposium, ESA SP-402

Høg, E. 1997b, in Procecdings of IAU Joint Discussion No. 14

Høg, E. et al. 1997, Astron. Astrophys. 323, L57

Kuzmin, A., Bastian, U., Høg, E., Kuimov, K., Röser, S. 1997, Tycho Reference Catalogue: Pilot Project Results, in Proceedings of the HIPPARCOS Venice ' 97 Symposium, ESA SP-402

Monet, D., et al. 1996, USNO-A V1.0, U.S. Naval Observatory, Washington

Yoshizawa, M., Andreasen, G.K., Høg, E., 1985, A\&A, 147, 227.

Röser, S., Bastian, U. 1991, PPM Star Catalogue, Astronomisches Rechen-Institut, Heidelberg. Spektrum Akademischer Verlag, Heidelberg, Berlin, New York

Röser, S., Høg, E. 1993, In: Workshop on Databases for Galactic Structure. Ed.: A.G. Davis Philip, B. Hauck and A.R. Upgren. Van Vleck Observatory Contr, No.13, 137. L. Davis Press, Schenectady, N.Y.

Röser, S., Morrison, J. Bucciarelli, B., Lasker, B. and McLean, B. 1996, In: IAU Symposium No. 179. Baltimore, USA, August, 26-30, 1996. (in press) 